\title{
A comparative study of Image Region-Based Segmentation Algorithms
}

\author{
Lahouaoui LALAOUI, \\ Laboratoiry LGE departement the elecronics University of \\ M'sila 28000 city Ichbilia, \\ M'sila, Algeria
}

\author{
Tayeb MOHAMADI, \\ Depart Electronics University Ferhat Abbas the Setif 19000 \\ city Elmaabouda. \\ M'sila, Algeria
}

\begin{abstract}
Image segmentation has recently become an essential step in image processing as it mainly conditions the interpretation which is done afterwards. It is still difficult to justify the accuracy of a segmentation algorithm, regardless of the nature of the treated image. In this paper we perform an objective comparison of region-based segmentation techniques such as supervised and unsupervised deterministic classification, non-parametric and parametric probabilistic classification. Eight methods among the well-known and used in the scientific community have been selected and compared. The Martin's(GCE, LCE), probabilistic Rand Index (RI), Variation of Information (VI) and Boundary Displacement Error (BDE) criteria are used to evaluate the performance of these algorithms on Magnetic Resonance (MR) brain images, synthetic MR image, and synthetic images. MR brain image are composed of the gray matter (GM), white matter (WM) and cerebrospinal fluid (CSF) and others, and the synthetic MR image composed of the same for real image and the plus edema, and the tumor. Results show that segmentation is an image dependent process and that some of the evaluated methods are well suited for a better segmentation.
\end{abstract}

Keywords-Evaluation criteria; Martin's; Rand Index; Image Segmentation; Magnetic resonance image.

\section{INTRODUCTION}

TRegion-based segmentation methods are powerful tools for objet detection and recognition. These methods aim at differentiating regions of interest (objects / background). Their objective is to divide the image into homogeneous zones to separate the different entities in the image. This is usually a first step in a more complex treatment chain involving pattern recognition. For example in medical imaging, segmentation is very important for representation and visualization as well as for the extraction of parameters and the analysis of images. Region based segmentation is a specific approach in which one seeks to construct surfaces by combining neighboring pixels according to a criterion of homogeneity. The nature of the considered images and the objective of the segmentation being multiple, there is no unique technique for image segmentation and segmenting an image into meaningful regions remains a real challenge [1]. According to Cocquerez et al.[2], the choice of a technique is related to the texture which is one of the important characteristics of an image. The purpose for based-region segmentation is to identify coherent regions of an image.

In order to compare the suitability of a segmentation method, we propose a comparative study between regions based segmentation techniques. To correctly validate a result of segmentation of medical images, it is necessary to have the ground truth, which is quite difficult in this case of real images. The quality of imagery and the requirement of accurate segmentation are the crucial aspect in characterizing the performance of segmentation algorithms in brain images [3],[4]. Many image processing techniques have been proposed for brain MRI segmentation, most notably thresholding [5], region-growing [6], classifying [7], clustering[8], modelling [9], neural network based [10] and others.

As can be seen on Error! Reference source not found., region based segmentation methods can be grouped into two famous families: deterministic based methods and probabilistic based classification methods. By the same way, each of these families can be subdivided into two groups. Deterministic classification family is composed of unsupervised and supervised methods. Whereas, probabilistic classification family contains parametric and non-parametric methods. In this paper, we present a comparative study of clustering based segmentation methods on synthetic and MR images. This paper is mainly devoted to study situations in which using different methods for the image segmentation. Its principal purpose is used five criteria and shows its suitability in unsupervised image segmentation. The performance of each technique is evaluated using the Martin's [11], Probabilistic Rand Index [12], Variation of Information [47] and Boundary Displacement Error criteria [53]. These measures compute the consistency degree between the regions produced by two segmentations. The evaluation of a segmentation algorithm consists in measuring the similarity between the reference algorithm and that obtained by this algorithm. The choice of an accurate measure is quite critical in order to provide a strict evaluation and reflect the real quality of an automatic segmentation with comparison to a manual one. The remainder of the paper is organized as follows: Section 2 presents the different region-based segmentation methods used for MR image analysis. The evaluation criteria are described in section 3. Section 4 describes the materiel and data used in this study. Experimental results on synthetic and real images are presented in section 5. Finally, a discussion concludes this paper in section 6 .

\section{REGION-BASED SEGMENTATION TECHNIQUES}

A large number of segmentation approaches have been proposed in the literature [13, 14, 15, 16]. A good survey about their evaluation can be found in [17][18]. A list of 


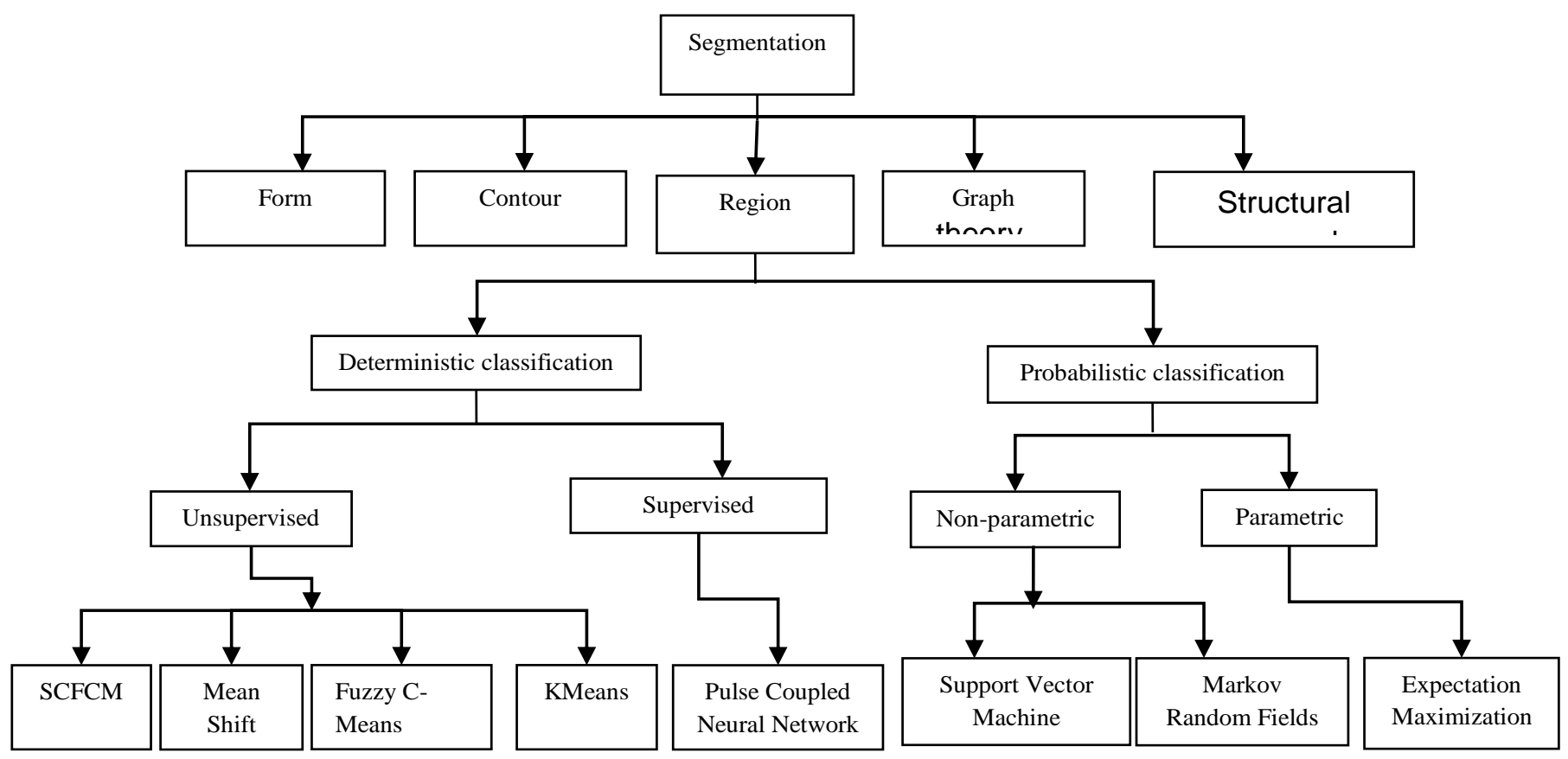

Fig. 1 Region-based segmentation methods

unsupervised, supervised, and non-parametric region based segmentation algorithms are presented in this section, such as Mean Shift (MS), Fuzzy C-Means (FCM), KMeans, Expectation Maximization (EM), Spatial Constraint Fuzzy CMeans (SCFCM), Markov Random Fields (MRF), Pulse Coupled Neural Network (PCNN), and Support Vector Machine (SVM). In the next subsections we will introduce briefly each of these techniques.

\section{A. K-Means}

K-Means algorithm is an unsupervised clustering algorithm that classifies the input data points into multiple classes based on their inherent distance from each other. The iterative K-Means clustering algorithm was first proposed by MacQueen [19]. The algorithm aims at partitioning the data set, consisting of $\ell$ expression patterns $\{\mathrm{x} 1, \ldots, \mathrm{x} \ell\}$ in an $\mathrm{n}$ dimensional space, into $\mathrm{k}$ disjoint clusters $\left\{\mathrm{C}_{\mathrm{i}}\right\}_{\mathrm{i}=1}{ }^{\mathrm{k}}$, such that the expression patterns in each cluster are more similar to each other than to the expression patterns in other clusters[20]. There are two popular partitioned clustering strategies: squareerror and mixture modeling. The sum of the squared Euclidian distances between the samples in a cluster and the cluster center is called within-cluster variation. K-Means are widely used in many applications such as data extraction and image segmentation [21]. The K-Means method is an iterative algorithm that minimizes the sum of distances between each object and its cluster centroid.

\section{B. Fuzzy C-Means (FCM)}

Fuzzy C-Means (FCM) is an unsupervised fuzzy clustering algorithm[22]. Excerpted from the algorithm of C-means[23], it introduces the concept of fuzzy set in the definition of classes, each point in the data set belongs to each cluster with a certain degree, and all clusters are characterized by their center of gravity. The FCM clustering algorithm was first suggested by Dunn [24] and later improved by Bezdek [25].The FCM method proposes a fuzzy membership that assigns a degree of membership for each class by iteratively updating the cluster centers and the membership degrees for each data point. The cluster that has an associated pixel is one whose membership degree is highest. A novel approach called enhanced possibilistic Fuzzy C-Means clustering is proposed for segmenting MR brain image into different tissue types on both normal and tumor affected pathological brain images. FCM methods has been proposed for the segmentation of MR Images [26,27]and for the segmentation of major tissues in $[28,29]$ and possible tumor on T1-weighted volumes. The FCM is often used in medical image segmentation [30, 31]. Chen et al. [32], have proposed an algorithm based on FCM for the correction of intensity in homogeneity and for segmentation of MRI images.

\section{Fuzzy C-Means algorithm with Spatial Constraint (SCFCM)}

Fuzzy C-Means algorithm with Spatial Constraint (SCFCM) is based on the clustering algorithm FCM described above, two kinds of information in image are used, the gray value, and space distributed structure. Based on the relevance of nearly pixels, the neighbors in the set should be similar in feature value. Its effectiveness contributes not only to introduction of fuzziness for belongingness of each pixel but also to exploitation of spatial contextual information. SCFCM clustering algorithm preserves the homogeneity of the regions better than existing FCM techniques, which often have difficulties when tissues have overlapping intensity. In order to reduce the noise effect during segmentation, the proposed 
method incorporates both the local spatial context and the nonlocal information into the standard FCM cluster algorithm using a novel dissimilarity index in place of the usual metric distance.

\section{Expectation Maximization (EM)}

Expectation Maximization (EM) is one of the most common algorithms used for density estimation of data points in an unsupervised setting. The EM algorithm [33]is used to estimate the parameters of this model; the resulting pixelcluster memberships provide a segmentation of the image. The EM algorithm can be considered as a variant of the K-Means algorithm where the membership of any given point to the clusters is not complete and can be fractional. An EM algorithm was proposed in [34]to model the homogeneities as a bias field of the image logarithm. This algorithm has been applied for the segmentation of brain MR image [35].According to [36]the EM algorithm has demonstrated greater sensitivity to initialization than the K-Means or FCM algorithms.

\section{E. Mean Shift (MS)}

The Mean Shift (MS) [37] algorithm clusters an ndimensional data set by associating each point with a peak of the data set's probability density. For each point, Mean Shift computes its associated peak by first defining a spherical window at the data point of radius $r$ and computing the mean of the points that lie within the window. At each iteration the window will shift to a more densely populated portion of the data set until a peak is reached, where the data is equally distributed in the window. MS was successfully applied by Mayer et al. [38]in clustering, segmentation and filtering of natural resources in 2D images [39], using a paradigm adaptively to segment the brain MR images.

\section{F. Markov Random Field (MRF)}

The Markov Random Field (MRF) models are used for the restoration and segmentation of digital images. They can make up for deficiencies in observed information by adding a-priori knowledge to the image interpretation process in the form of models of spatial interaction between neighboring pixels. Hence, the classification of a particular pixel is based, not only on the intensity of that pixel, but also on the classification of neighboring pixels. The goal of segmentation is to estimate the correct label for each site. The segmentation is obtained by classifying the pixels into different pixel classes. These classes are represented by multivariate Gaussian distributions. A most of reference are cited, It can be viewed as a particular model selection problem, and different techniques have been proposed in the classical HMF case [40]. It has been used for brain image segmentation by modeling probabilistic distribution of the labeling of a voxel jointly with the consideration of the labels of a neighborhood of the voxel [41].

\section{G. Support vector machine (SVM)}

The Support Vector Machine (SVM) is a learning machine for two-group classification problems.

The machine conceptually implements the following idea: input vectors are non-linearly mapped to a very high- dimension feature space. SVM is a set of supervised learning techniques for solving problems of discrimination, regression and are particularly adapted to data process at very high dimensions [42]. The algorithm of the SVM is described as follows:

First specifies a small set of training pixels, such as a small part of an object and a small part of the background, as the clues. Then, fast SVM is applied to train the classifiers based on the training pixels. Finally, the remaining image, which is viewed as the test set, is subdivided into several regions by the classifier. A comparison between a segmentation method with SVM and FCM is applied in [43].

\section{H. The Pulse-Coupled Neural Network (PCNN)}

The Pulse-Coupled Neural Nets (PCNN) is a twodimensional non-training neural network in which each neuron in the network corresponds to one pixel in an input image. The neuron receives its input as an external stimulus. These stimuli are combined in an internal activation system, and are accumulated until they exceed a dynamic threshold. This will result in a pulse output and through an iterative process. The algorithm produces a temporal series of binary images as outputs algorithm is based on the neurophysiologic models evolving from studies of small mammals. Depending on time as well as on the parameters, this dynamic output contains information, which makes it possible to detect edges, do segmentation, identify textures and perform other feature extractions. For the PCNN, the neurons associated with each group of spatially connected pixels with similar intensities tend to pulse together [44]. This is the basic principle of segmentation of the PCNN. In fact, there are many approaches for image segmentation with the PCNN. Generally, all the methods of segmentation can be classified into two kinds of schemes: common image segmentation and automatic image segmentation.

\section{EVALUATION CRITERIA}

The goal of this study is to perform a quantitative comparison between automatic segmentation and a set of ground truth segmentation (reference). We use the same methodology reported in, and an evaluation metric for image segmentation of multiple objects [45], where a quantitative predictive performance evaluation used full reference image quality assessment metrics has been conducted. In this section we present five criteria, the Probabilistic Rand Index, Global Consistency Error, Local Consistency Error, Boundary Displacement Error and Variation of Information.

\section{A. The Probabilistic Rand Index (PRI)}

In literature there are many criteria of nonparametric measures such as: Jaccard's index, Fowlkes, and Mallow's index [46], he is work by counting pairs of pixels that have compatible label relationships between the two segmentations to be compared. We consider two images reference and segmented respectively $\mathrm{S} 1$ and $\mathrm{S} 2$ of $\mathrm{N}$ points $\mathrm{X}=\left\{x_{1}, x_{2}, x_{3}\right.$, $\left.x_{4}, \ldots, x_{N}\right\}$; that assigned labels $\left\{b_{i}\right\}$ and $\left\{b_{i}^{\prime}\right\}$ respectively to point xi. The Rand Index can be computed as the ratio of the number of pairs of vertices or faces having the compatible label relationship in $\mathrm{S} 1$ and $\mathrm{S} 2$. Can be defined as: 


$$
\begin{aligned}
R\left(S_{1}, S_{2}\right)=\frac{1}{\left(2^{N}\right)} \sum_{\substack{i, j \\
i \neq j}}\left[I\left(l_{i}=l_{j} \wedge l_{i}^{\prime}=l_{j}^{\prime}\right)\right. \\
\left.+I\left(l_{i} \neq l_{j} \wedge l_{i}^{\prime} \neq l_{j}^{\prime}\right)\right]
\end{aligned}
$$

Where I is the identity function, and the denominator is the number of possible unique pairs among $\mathrm{N}$ data points. This gives a measure of similarity ranging from 1, when the two images reference and segmented respectively are identical, to 0 other wise. We first outline a generalization to the Rand Index, termed the Probabilistic Rand (PR) index, which we previously introduced in [47] The PR index allows comparison of test segmentation with multiple ground-truth images through soft non uniform weighting of pixel pairs as a function of the variability in the ground-truth set. The Rand index [47] counts the fraction of pairs of pixels whose labeling are consistent between the computed segmentation and the ground truth. This quantitative measure is easily extended to the probabilistic Rand index (PRI) [48] by averaging the result across all human segmentations of a given image. Consider a set of manually segmented (ground truth) images $\{\mathrm{S} 1, \mathrm{~S} 2, \ldots$, $\mathrm{SK}\}$ corresponding to an image $\mathrm{X}=\{\mathrm{x} 1, \mathrm{x} 2, \ldots \mathrm{x} i, \ldots, \mathrm{xN}\}$, where a subscript indexes one of $\mathrm{N}$ pixels. Let $\mathrm{S}$ test be the segmentation that is to be compared with the manually labeled set.

\section{B. Martin Evaluation Criteria}

Martin et al.[49] proposed two error measures to quantify the consistency between image segmentations of differing granularities, and used them to compare the results of algorithms to a database of manually segmented images. The Martin's similarity index which outperforms the others in terms of properties and discriminative power is employed for performance evaluation to compare the different region-based segmentation methods. The role of the test is to assess the quality of segmentation by transforming the measurements into a mathematical function called test. These criteria may be a test of homogeneity of a set of points of similarity, or any statistical test. Martin et al. [50] proposed an interesting error measure, which takes 2 images $\mathrm{S} 1$ and $\mathrm{S} 2$ as input, and produces a real-valued output in the range [0, 1], the Martin's distance where 0 signifies no error and 1 worst segmentation, which the inverse for similarity 1 signifies no error and 0 worst segmentation. The measure is shown to be effective for qualitative similarity comparison between segmentations by humans, who often produce results with varying degrees of perceived details, which are all intuitively reasonable and therefore "correct". On the other hand, the Martin error measure is sensitive to qualitatively different segmentations. A segmentation error measure takes two segmentations $\mathrm{S} 1$ and $\mathrm{S} 2$ as input, and produces a real valued output. For a given pixel pi consider the segments in S1 and S2 that contain that pixel. The segments are sets of pixels. If one segment is a proper subset of the other, then the pixel lies in area of refinement and the local error should be zero. If there is no subset relationship, then the two regions overlap in an inconsistent manner. In this case, the local error should be non-zero. If $\mathrm{R}(\mathrm{S}, \mathrm{pi})$ is the set of pixels corresponding to the region in segmentation $S$ which is the region that contains pixels pi, the local refinement error, $\mathrm{E}$, is defined as:
$E\left(S_{1}, S_{2}, p_{i}\right)=\frac{\left|R\left(S_{1}, p_{i}\right) / R\left(S_{2}, p_{i}\right)\right|}{\left|R\left(S_{1}, p_{i}\right)\right|}$

Note that this local error measure is not symmetric. It encodes a measure of refinement in one direction only: $E\left(S_{1}, S_{2}, p_{i}\right)$ is zero precisely when $\mathrm{S} 1$ is a refinement of $\mathrm{S} 2$ at pixel pi, but not vice versa. There are two natural ways to combine the values into a measure of the error for the entire image. Global Consistency Error (GCE) forces all local refinements to be in the same direction. Local Consistency Error (LCE) allows refinement in different directions and in different parts of the image. Let $\mathrm{n}$ be the number of pixels:

$$
\begin{gathered}
\operatorname{GCE}\left(\mathrm{S}_{1}, \mathrm{~S}_{2}\right)=\frac{1}{\mathrm{n}} \min \left\{\sum_{\mathrm{i}} \mathrm{E}\left(\mathrm{S}_{1}, \mathrm{~S}_{2}, \mathrm{p}_{\mathrm{i}}\right), \sum_{\mathrm{i}} \mathrm{E}\left(\mathrm{S}_{2}, \mathrm{~S}_{1}, \mathrm{p}_{\mathrm{i}}\right)\right\} \\
\operatorname{LCE}\left(\mathrm{S}_{1}, \mathrm{~S}_{2}\right)=\frac{1}{\mathrm{n}} \sum_{\mathrm{i}} \min \left\{\mathrm{E}\left(\mathrm{S}_{1}, \mathrm{~S}_{2}, \mathrm{p}_{\mathrm{i}}\right), \mathrm{E}\left(\mathrm{S}_{2}, \mathrm{~S}_{1}, \mathrm{p}_{\mathrm{i}}\right)\right\}
\end{gathered}
$$

Although these error metrics are calculated by grouping pixels into objects first, they unfortunately tolerate oversegmentation and under-segmentation, as a consequence of their intended purpose for comparing human segmentations. As $\mathrm{LCE} \leq \mathrm{GCE}$, it is clear that GCE is a tougher measure than LCE.

\section{Boundary matching (Boundary Displacement Error)}

Several measures work by matching boundaries between the segmentations, and computing some summary statistic of match quality. The Boundary Displacement Error (BDE) measures the average displacement error of one boundary pixels and the closest boundary pixels in the other segmentation [48]. Work in [51] proposed solving an approximation to a bipartite graph matching problem for matching segmentation boundaries, computing the percentage of matched edge elements, and using the harmonic mean of precision and recall, termed the F-measure as the statistic. Furthermore, for a given matching of edge elements between two images, it is possible to change the locations of the unmatched edges almost arbitrarily and retain the same precision and recall score.

\section{Information-based (Variation of Information)}

The proposed metric measure is termed the variation of information (VI) and is related to the conditional entropies between the class label distributions of the segmentations. Work in [52] computes a measure of information content in each of the segmentations and how much information one segmentation gives about the other. Several measures work by counting the number of false- positives and false-negatives [53] and similarly assume existence of only one ground truth segmentation. Due to the lack of spatial knowledge in the measure, the label assignments to pixels may be permuted in a combinatorial number of ways to maintain the same proportion of labels and keep the score unchanged.

\section{MATERIEL}

\section{A. Data synthetic MR image}

A large number of segmentation approaches have been proposed in the literature $[54,55,56,57]$. A good survey about their evaluation can be found in [58][59] A list of unsupervised, supervised, and non-parametric region based 
segmentation algorithms are presented in this section, such as Mean Shift (MS), Fuzzy C-Means (FCM), KMeans, Expectation Maximization (EM), Spatial Constraint Fuzzy CMeans (SCFCM), Markov Random Fields (MRF), Pulse Coupled Neural Network (PCNN), and Support Vector Machine (SVM). The image is constitute with: White matter(WM), gray matter (GM), cerebrospinal fluid(CSF), edema, tumor for the synthetic MR image are represented as a set of spacial probability maps for tissue and pathology shows in Figure.2. In our laboratory, from these different matters we have created the ground truth for each image.
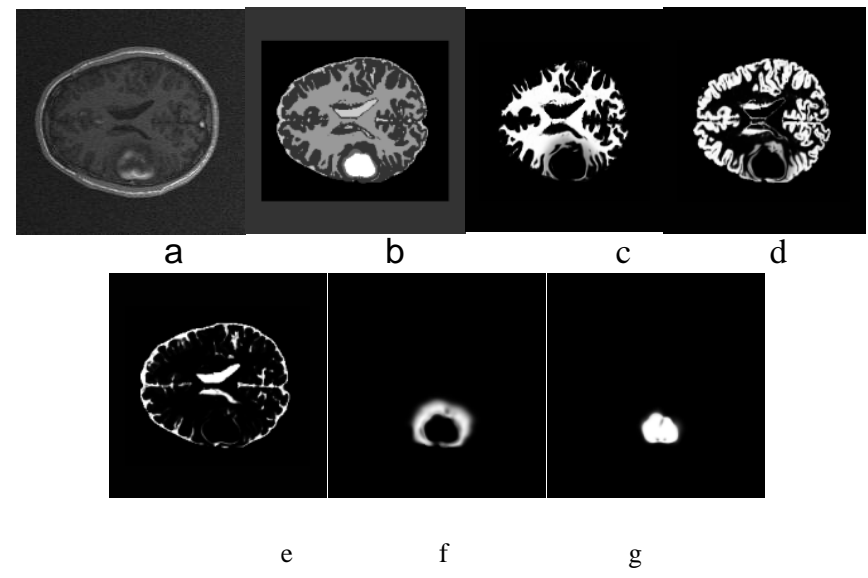

Fig. 2 From left to right: (a) image T1, (b) ground truth, (c) white matter, (d) gray matter, (e) CSF, (f) edema, (g) tumor.

In this section we compare the results of segmentation methods on synthetic MR image. In Fig 2 present an example of synthetic MR image with the display the images of different segmentation methods. And in the Table 2 below shows the values of evaluation criteria. These examples we allow to understand how to meet these criteria have different images segmentation. The values obtained in Table 2 shows the comparison between the automatic image segmentation and the ground truth image for synthetic MR images. The results of average and variance we applied for 25 synthetic MR image the results are given in following are varies between $0.3245 \pm 0.0012$ and $0.5021 \pm 0.0013$ for GCE criterion, the value between $0.124 \pm 0.0034$ and $0.3585 \pm 0.0070$ for LCE, the values between $0.4069 \pm 0.0058$ and $0.5912 \pm 0.0067$ for PRI, the values between $1.5021 \pm 0.5871$ and $5.2314 \pm 1.2341$ for VI, and $92.8908 \pm 22.5487$ and $3.7077 \pm 0.6532$ for BDE criterion.

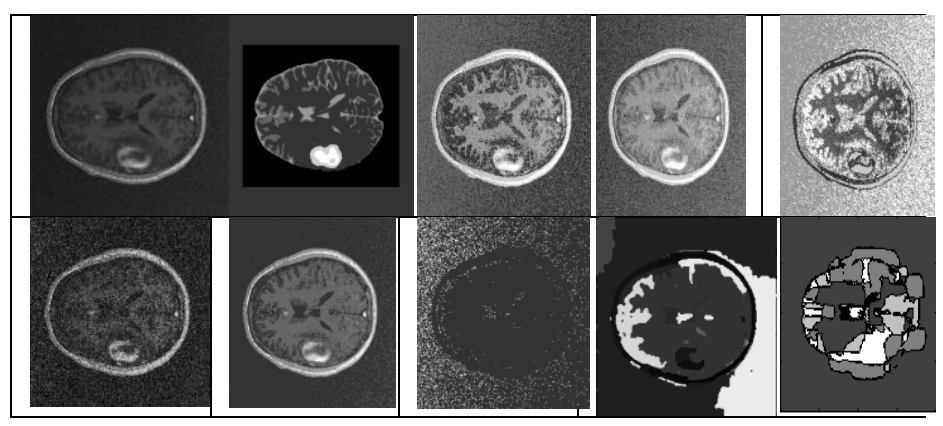

Fig. 3 Representative examples of results obtained with the different segmentation methods, (a) original image, (b) ground truth image, (c) FCM, (d) K-Means, (e) SCFCM, (f) MS, (g) EM, (h) MRF,(i) PCNN (j) SVM.

\section{B. Real data}

In this section, images are obtained from the IBSR (Internet Brain Segmentation Repository) database [60]. As described on the IBSR, the database is composed of threedimensional coronal brain Magnetic Resonance Images (MRI). The coronal three-dimensional T1-weighted spoiled gradient echo MRI scans were performed on 2 different imaging systems.

The MR Brain data sets and their manual segmentations were provided by the center of morphometric analysis at Massachusetts general hospital and are available at IBSR. The voxels contain images segmented by experts for each subdatabases are the ground truth voxels. These databases are used by many that users' all around the world. It supplies brain MR images as well as the segmentation results that are performed by the trained experts in a manually guided manner. Error! Reference source not found.3shows different images from the IBSR database. For our experiment, we used 25 test images from the IBSR database and the corresponding ground truth (segmented by the expert) to each image.

The different based segmentation methods are applied on each image and the Martin's criteria are used to evaluate the performance of each algorithm.
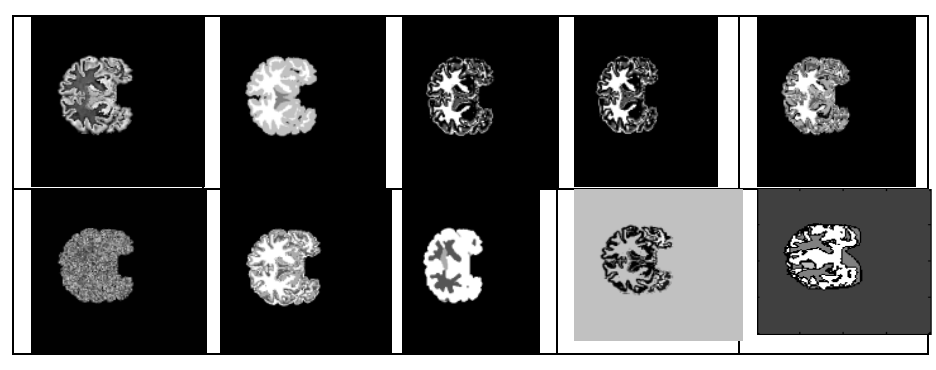

Fig. 4 Representative examples of results obtained with the different segmentation methods, (a) original image, (b) ground truth image, (c) FCM, (d) K-Means, (e) SCFCM, (f) MS, (g) EM, (h) MRF,(i) PCNN (j) SVM.

The analysis of the results of Fig. 4 demonstrates that some of the used algorithms generate as many classes as those generated by the laboratory. These findings are confirmed by the criteria reported in Table 3.

Comparison of the eight segmentation algorithms using LCE and GCE errors (mean) in the case of real images in these paper 25 images. The MS method performs better than the FCM, followed respectively by SVM, SCFCM, EM, K-Means, MRF, and PCNN. Accordingly, we compare the segmentation performance in brain tissue. To say that actual results are consistent with the results obtained on synthetic images.

For a quick interpretation of the results, Fig. 4 report the evolution of the martin's criteria. The best criterion values are obtained for the EM method (GCE criterion $=0.9268$, LCE criterion $=0.9047, \mathrm{PRI}=0.9724, \mathrm{VI}=0.4935$, and $\mathrm{BDE}=$ 3.245 ) in average. 
TABLE I. Averages and STD of: GCE, LCE, PRI, VI, and BDE mean values of the synthetic MR Dataset for the different segmentation methods.

\begin{tabular}{|c|c|c|c|c|c|c|c|c|c|}
\hline criteria & & FCM & K-Means & SCFCM & MS & EM & MRF & PCNN & SVM \\
\hline \multirow{2}{*}{ GCE } & Average & 0.4152 & 0.4090 & 0.3795 & 0.3397 & 0.5021 & 0.4040 & 0.4021 & 0.3245 \\
\hline & $\begin{array}{l}\text { STD } \\
\end{array}$ & 0.0052 & 0.0049 & 0.0050 & 0.0064 & 0.0013 & 0.0062 & 0.0063 & 0.0012 \\
\hline \multirow{2}{*}{ LCE } & Average & 0.3569 & 0.3443 & 0.1941 & 0.3149 & 0.3585 & 0.1284 & 0.3054 & 0.124 \\
\hline & STD & 0,0041 & 0,0071 & 0,0564 & 0,0083 & 0,0070 & 0.0017 & 0,0064 & 0.0034 \\
\hline \multirow{2}{*}{ PRI } & Average & 0.5882 & 0.5826 & 0.5912 & 0.5552 & 0.5771 & 0.4165 & 0.4069 & 0.502 \\
\hline & STD & 0.0077 & 0.0066 & 0.0067 & 0.045 & 0.0088 & 0.0062 & 0.0058 & 0.0084 \\
\hline \multirow[t]{2}{*}{ VI } & Average & 3.1525 & 3.0997 & 3.2087 & 3.5489 & 2.3710 & 1.5021 & 1.5845 & 5.2314 \\
\hline & STD & 0.9154 & 0.885 & 0.456 & 0.658 & 0.451 & 0.5871 & 0.1854 & 1.2341 \\
\hline \multirow{2}{*}{ BDE } & Average & 4.6365 & 4.7548 & 4.5193 & 4.5782 & 3.7077 & 92.8908 & 53.3238 & 24.2145 \\
\hline & STD & 0.9124 & 0.7245 & 0.8546 & 0.8546 & 0.6532 & 22.5487 & 16.2354 & 5.123 \\
\hline criteria & & FCM & K-Means & SCFCM & MS & EM & MRF & PCNN & SVM \\
\hline \multirow{2}{*}{ GCE } & Average & 0.4152 & 0.4090 & 0.3795 & 0.3397 & 0.5021 & 0.4040 & 0.4021 & 0.3245 \\
\hline & $\begin{array}{l}\text { STD } \\
\end{array}$ & 0.0052 & 0.0049 & 0.0050 & 0.0064 & 0.0013 & 0.0062 & 0.0063 & 0.0012 \\
\hline \multirow{2}{*}{ LCE } & Average & 0.3569 & 0.3443 & 0.1941 & 0.3149 & 0.3585 & 0.1284 & 0.3054 & 0.124 \\
\hline & $\begin{array}{l}\text { STD } \\
\end{array}$ & 0,0041 & 0,0071 & 0,0564 & 0,0083 & 0,0070 & 0.0017 & 0,0064 & 0.0034 \\
\hline \multirow{2}{*}{ PRI } & Average & 0.5882 & 0.5826 & 0.5912 & 0.5552 & 0.5771 & 0.4165 & 0.4069 & 0.502 \\
\hline & STD & 0.0077 & 0.0066 & 0.0067 & 0.045 & 0.0088 & 0.0062 & 0.0058 & 0.0084 \\
\hline \multirow[t]{2}{*}{ VI } & Average & 3.1525 & 3.0997 & 3.2087 & 3.5489 & 2.3710 & 1.5021 & 1.5845 & 5.2314 \\
\hline & STD & 0.9154 & 0.885 & 0.456 & 0.658 & 0.451 & 0.5871 & 0.1854 & 1.2341 \\
\hline \multirow{2}{*}{ BDE } & Average & 4.6365 & 4.7548 & 4.5193 & 4.5782 & 3.7077 & $\begin{array}{l}92.8908 \\
\end{array}$ & 53.3238 & 24.2145 \\
\hline & STD & 0.9124 & 0.7245 & 0.8546 & 0.8546 & 0.6532 & 22.5487 & 16.2354 & 5.123 \\
\hline
\end{tabular}

TABLE II. Averages and STD of: GCE, LCE, PRI, VI, and BDE mean values of the dataset for the different segmentation methods.

\begin{tabular}{|l|l|l|l|l|l|l|l|l|l|}
\hline criteria & & FCM & KMeans & SCFCM & MS & EM & MRF & PCNN & SVM \\
\hline \multirow{2}{*}{ GCE } & Mean & 0.9161 & 0.9169 & 0.7922 & 0.9180 & 0.9268 & 0.9028 & 0.8480 & 0.7856 \\
\cline { 2 - 9 } & STD & 0.0275 & 0.0285 & 0.1604 & 0.0317 & 0.0263 & 0.0210 & 0.0491 & 0.0210 \\
\hline \multirow{2}{*}{ LCE } & Means & 0.0829 & 0.8872 & 0.2308 & 0.8893 & 0.9047 & 0.8872 & 0.8893 & 0.7544 \\
\cline { 2 - 10 } & STD & 0.0117 & 0.0203 & 0.3141 & 0.0141 & 0.0154 & 0.0125 & 0.0141 & 0.0107 \\
\hline \multirow{2}{*}{ PRI } & Mean & 0.9315 & 0.9301 & 0.9221 & 0.6815 & 0.9724 & 0.9768 & 0.84786 & 0.8324 \\
\cline { 2 - 10 } & STD & 0.0164 & 0.0172 & 0.0083 & 0.0525 & 0.0093 & 0.0075 & 0.0248 & 0.0650 \\
\hline \multirow{2}{*}{ VI } & Mean & 0.6637 & 0.6668 & 0.7014 & 0.8679 & 0.4935 & 0.5478 & 0.7581 & 0.6847 \\
\cline { 2 - 10 } & STD & 0.1255 & 0.1280 & 0.0465 & 0.1141 & 0.1096 & 0.1245 & 0.0145 & 0.1128 \\
\hline \multirow{2}{*}{ BDE } & Mean & 0.6806 & 0.6731 & 0.7000 & 82.2160 & 3.245 & 8.5471 & 0.624 & 18.7584 \\
\cline { 2 - 9 } & STD & 0.0924 & 0.0943 & 0.0436 & 4.0190 & 0.8096 & 1.1211 & 0.0074 & 8.0874 \\
\hline
\end{tabular}


Table 3 shows the output of the criteria; interval with a lower limit greater than 0 and high limited at 1 , the values implies that the adaptive EM performs significantly better in segmentation than benchmark (the FCM, K-Means, SCFCM, MS, MRF, PCN or SVM). The GCE, LCE, and RI values of the EM method in Fig 12, for 25 brain images, which demonstrate the robustness of the method EM.

TABLE III. CPU time by different algorithms in Fig.11 in the same order.

\begin{tabular}{|l|c|c|c|}
\hline & $\begin{array}{l}\text { Image 256×256 } \\
\text { pixels } \\
524288 \text { bytes }\end{array}$ & $\begin{array}{l}\text { Image 180x180 } \\
\text { pixels } \\
259200 \text { bytes }\end{array}$ & $\begin{array}{l}\text { Image } \\
172 \times 158 \\
\text { pixels } \\
217408 \\
\text { bytes }\end{array}$ \\
\hline FCM (CPU time(s)) & 12.11 & 5.76 & 4.09 \\
\hline $\begin{array}{l}\text { K-Means (CPU } \\
\text { time(s)) }\end{array}$ & 2.27 & 1.82 & 1.01 \\
\hline $\begin{array}{l}\text { SCFCM (CPU } \\
\text { time(s)) }\end{array}$ & 23.41 & 10.45 & 7.46 \\
\hline MS (CPU time(s)) & 0.35 & 0.46 & 0.39 \\
\hline EM (CPU time(s)) & 20.86 & 9.08 & 7.38 \\
\hline MRF (CPU time(s)) & 1470.88 & 673.17 & 564.15 \\
\hline PCNN (CPU time(s)) & 10.76 & 6.09 & 6.05 \\
\hline SVM (CPU time(s)) & 18.12 & 8.21 & 7.25 \\
\hline
\end{tabular}

4.5. Computational time

The processing time for segmenting images is presented in Table 4. We list the CPU time in segmenting images in Fig.10. It can be seen from Table 4 that the processing time for MRF are both higher than the other algorithms.

\section{DisCUSSION AND CONCLUSION}

This paper presents an objective comparison of regionbased segmentation methods. Our study focuses on supervised and unsupervised deterministic classification, non-parametric and parametric methods probabilistic classification. Among the well-known and used techniques in the scientific community, we have selected eight techniques. These methods have been used on two different databases. The first composed synthetic MR images are available for download at www.ucinia.org, and the second composed of brain MR images from the IBSR database. For comparison, a ground truth is created in our laboratory for synthetic MR images and by an expert for IBSR database. To compare the different region based segmentation methods, we used the Martin's similarity indexes and Probabilistic Rand Index. Five criteria have been used: The global consistency error, the local consistency error, Probabilistic Rand Index, Variation of Information, and Boundary Displacement Error. At each time, the result of these criteria is the difference between the automatic segmentation and the ground truth. In this paper, we compared the performance of different region-based segmentation algorithms. Results show that the EM is outperforms the other seven algorithms in the three different dataset images. The analysis of the results of the five criteria demonstrate that except the EM, K-Means, SCFCM and the FCM algorithm, all the methods that we have tested perform well for the segmentation of images such those considered in this paper. Nevertheless, we are going to group them in two classes. The first class contained SCFCM, K-Means, FCM, and EM, the latter algorithm has a best performance with $\mathrm{GCE}=0.6935, \quad \mathrm{LGE}=0.4113$ and $\mathrm{PRI}=0.8245$ for the synthetic data, $\mathrm{GCE}=0.5021, \mathrm{LGE}=0.3585$ and $\mathrm{PRI}=0.6067$ for the synthetic MR data, and with $\mathrm{GCE}=0.9268$, $\mathrm{LGE}=0.9047$, and PRI $=0.6067$ for the ISBR data. This is consistent with what have been reported on the robustness of the MS algorithm for feature extraction and image segmentation. The MS algorithm is an unsupervised clustering-based segmentation method and needs no a priori information on the number and the shape of the data cluster. The FCM method takes advantage of local textual information and high inter-pixel correlation inherent. The second class, with a worst quality scores for the criteria groups decreasing: MRF, MS, PCNN, and SVM methods. The very high value of the five criteria for the EM method is due to known fixed segmentation parameters of the EM method estimated by optimizing the likelihood. The optimized requires no 'step size' parameters and will not oscillate around the optimum. However, there is no guarantee of global solutions. These results might be due to initialization the parameter for each algorithm. Last but not least, according to Error! Reference source not found. which reports the least values obtained for the GCE, LCE, PRI, VI, and BDE on the synthetic data it is shown that the demonstrated EM method is well adapted for any type of images synthetic MR, and MR images. In the second, by the FCM, K-Means, and SCFCM methods almost the same values of five criteria in different type of the three datasets. In this paper, the adaptive EM is outperforms the other seven algorithms in three dataset (synthetic MR images, and MR images).As a prospect to this study, we are actively working on $3 \mathrm{D}$ segmentation methods. In progress as well, a study to compare criteria for evaluation of the image segmentation methods.

\section{References}

[1] G. R. Dattatreya, "Unsupervised context estimation in a mesh of pattern classes for image recognition", Pattern Recognition, Vol. 24(7), pp. 685694, 1991.

[2] Cocquerez et al, Analyse d'images : filtrage et segmentation, Ed Masson, 1995.

[3] Herng-Hua Chang, Performance measure characterization for evaluating neuro image segmentation algorithms, NeuroImage 47 (2009) 122-135

[4] Jifeng Ning et al. Interactive image segmentation by maximal similarity based region merging, Pattern Recognition 43 (2010) 445 - 456.

[5] Salem Saleh Al-amri, N.V. Kalyankar and Khamitkar S.D , " Image Segmentation by using Thershod Techniques", Journal of Computing,vol2, pp 83-86,2010

[6] R. Pohle and K. D. Toennies, "Segmentation of medical images using adaptive region growing," Proc. SPIE - Med. Imag., vol. 4322, pp. 1337-1346, 2001.

[7] R.J. Schalkoff. Pattern recognition: statistical, structural and neural approaches. John Wiley and Sons, 1992.

[8] S. Shen, W. A. Sandham, M. H. Grant, J. Patterson, and M. F. Dempsey, "Fuzzy clustering based applications to medical image processing," in Proc. IEEE EMBS 25th Annu. Int. Conf., 2003, pp. 747-750.

[9] S.Z. Li. Markov random field modeling in computer vision. Springer, 1995.

[10] L.O. Hall, A.M. Bensaid, L.P. Clarke, R.P. Velthuizen, M.S. Silbiger, and J.C. Bezdek. A comparison of neural network and fuzzy clustering techniques in segmenting magnetic resonance images of the brain. IEEE T. Neural Networks, 3:672-682, 1992.

[11] Martin, D., Fowlkes, C., Tal, D., Malik, J., 2001. A database of human segmented natural images and its application to evaluating algorithms and measuring ecological statistics, ICCV (2001) 416 - 423.

[12] R. Unnikrishanan M. Hebert, "measures of similarity," proc. IEEE workshop computer vision applications, 2005. 
[13] yongxia et all, image segmentation by clustering of special patterns, pattern recognition letters, 28(2007) 1548-1555.

[14] Kannan, S.R., 2008. A New segmentation system for Brain MR images based on fuzzy techniques. J. Appl. Soft Comput. 8(4), 1599-1606.

[15] Zenaty, E.A., Aljahdali, S., Debnath, N., 2009. A Karnalized Fuzzy CMeans algorithm for automatic MRI segmentation. Comput. Methods Sci.Eng. 9, 123-136, doi 10.3233/JCM-2009-0241.

[16] Chuang. K.,- S., et al., 2006.fuzzy C-Means clustering with spatial information for image segmentation. Comput.Med. Imaging Graph.3,915.

[17] H. Zhang, J. E. Fritts, Image segmentation evaluation: A survey of unsupervised methods, computer vision and image understanding 110 (2008) 260-280

[18] S.Rian, C. Jqggim J. Xue, 'Brain Tissue classification of magnetic Resonance images using partial volume modeling', IEEE Trans. On medical imaging, vol.19, No.2, pp.1179-1187, 2000.

[19] S. K. Khan and A. Ahmad. Cluster center initialization algorithm for kmeans clustering. Pattern Recognition Letters, 25:1293-1302, 2004.

[20] M. Mignotte. Segmentation by fusion of histogram-based k-means clusters in different color spaces. IEEE Transactions on Image Processing, 17(5):780-787, 2008.

[21] Zhang, Y. J. (2002a). "Image engineering and related publications" International Journal of Image and Graphics, (2002) 2(3), 441-452.

[22] Pham D.L and Prince J.L. An adaptive fuzzy C-means algorithm for image segmentation in the presence of intensity inhomogeneities. Pattern Recognition Letters. vol. 20, no.1, pp.57-68, 1999

[23] Richard J. Hathaway, John W. Davenport and James C. Bezdek 'Relational duals of the c-means clustering algorithms' Pattern Recognition, Volume 22, Issue 2, 1989, Pages 205-212.

[24] J. C. Dunn (1973): "A Fuzzy Relative of the ISODATA Process and Its Use in Detecting Compact Well-Separated Clusters", Journal of Cybernetics 3: 32-57.

[25] J. C. Bezdek, J. M. Keller, R. Krishnapuram, and N. R. Pal, Fuzzy Models and Algorithms for Pattern Recognition and Image Processing. New York: Springer, 1999.

[26] Claramunt CF., Sede M.H., Prelaz-Droux R., Vidale L., « Sémantique et logique spatio-temporelles ». Revue internationale de géomatique, volume 4, pp. 165-180, 1994.

[27] R.L. Cannon, J.V. Dave, J.C. Bezdek. - Efficient implementation of the fuzzy c-mean clustering algorithms. IEEE Transaction on Pattern Analysis and Machine Intelligence, vol. 8, n2, 1986, pp. 248-255.

[28] Songcan Chen and Daoqiang Zhang. Robust Image Segmentation Using FCM With Spatial Constraints Based on New Kernel-Induced Distance Measure. IEEE transactions onsystems, man, and cybernetics-part b: cybernetics, vol. 34, no. 4, august, 2004.

[29] S. Ruan, B. Moretti, J. Fadili, and D. Bloyet. Fuzzy Markovian Segmentation in Application of Magnetic Resonance Images. Computer Vision and Image Understanding, 85:54-69, 2002.

[30] D. Pham \& J. L. Prince. Adaptative Fuzzy Segmentation of Magnetic Resonance Images. IEEE Transactions on Medical Imaging, vol. 18, no. 9, pages 737-752, September 1999.

[31] S. Shen, W. Sandham, M. Granat \& A. Sterr. MRI Fuzzy Segmentation of Brain Tissue Using Neighborhood Attraction With Neural-Network Optimization. IEEE Transactions on Information Technology In Biomedicine, vol. 9, no. 3, pages 459-467, September 2005.

[32] Weijie Chen, Maryellen L. Giger. - A fuzzy c-means (FCM) based algorithm for intensity in homogeneity correction and segmentation of MR images. In : Proceedings of IEEE International Symposium on Biomedical Imaging, pp. 1307-1310, Arlington, VA, USA, 2004.

[33] A. Dempster, N. Laird, and D. Rubin, Maximum Likelihood from Incomplete Data via the EM Algorithm, J. Royal Statistical Soc., Ser. B, vol. 39, no. 1, pp. 1-38, 1977.

[34] W. M. Wells, W. E. L. Grimson, R. Kikins, and F. A. Jolesz, "Adaptive segmentation of MRI data," IEEE Trans. Med. Imag., vol. 15, pp. 429442, Aug. 1996.
[35] K. V. Leemput, F. Maes, D. Vandermeulen, and P. Suetens, "Automated model-based tissue classification of MR images of the brain," IEEE Trans. Med. Imag., vol. 18, no. 10, pp. 897-908, Oct. 1999.

[36] Z.Yu, O.C, Au,R.Zou, W. Yu, J. Tian, an adaptative unsupervised approach toward pixel clustering and color image segmentation. Pattern recognition. 43(5)(2010)1889-1906.

[37] D. Comaniciu and P.Meer,"Mean Shift: A robust approach toward feature space analysis", IEEE Trans. Pattern Analysis and Machine Intelligence, Vol. 24, No. 5, May 2002, pp. 603-619

[38] A. Mayer et H. Green span: Segmentation of brain MRI by adaptive mean shift. International Symposium on Biomedical Imaging: Macro to Nano, pages 319-322, avril 2006.

[39] Comaniciu, D., Meer, P.: Mean shift: a robust approach toward feature space analysis. Pattern Analysis and Machine Intelligence, IEEE Transactions on 24(5) (May 2002) 603-619

[40] F. Forbes, N. Peyrard, Hidden Markov random field model selection criteria based on mean field-like approximations, IEEE Trans. Pattern Anal. Mach. Intell. 25 (9) (2003) 1089-1101.

[41] Ali, A.A., Dale, A.M., Badea, A, Johnson, G.A., 2005. Automated segmentation of neuroanatomical structures in multispectral MR microscopy of the mouse brain, NeuroImage 27 (2), 425-435.

[42] V. N. Vapnik, Statistical Learning Theory, New York, wiley, édition, 1998.

[43] C. Bielski and P. Soille. Order independent image compositing. Lecture Notes in Computer Science, 3617:1076-1083, September 2005.

[44] Murali Murugavel, John M, Sullivan. Jr, 'Automatic cropping of MRI rat brain volumes using pulse coupled neural networks', NeuroImage 45 (2009) 845-854.

[45] Marak Polak, Hong Zhang, An evaluation metric for image segmentation of multiple objects, Image and Vision Computing. Image and Vision Computing. 27 (2009) 1223-1227.

[46] Fowlkes, E.B., Mallows, C.L.: A method for comparing two hierarchical clustering. J. Am. Stat. Assoc. 78(383), 553-569 (1983).

[47] R. Unnikrishnan, C. Pantofaru, and M. Hebert, , A Measure of objective evaluation of image segmentation algorithms," in Proc. IEEE Conf. Computer Vision and Pattern Recognition Workshop on Empir- ical Evaluation Methods in Computer Vision, Jun. 2005, vol. 3, pp. 34-41.

[48] R. Unnikrishnan, C. Pantofaru, and M. Hebert, "Toward objective evaluation of image segmentation algorithms," IEEE Trans. Pattern Anal. Mach. Intell., vol. 29, no. 6, pp. 929-944, Jun. 2007.

[49] D. Martin, "An Empirical approach to grouping and segmentation", Ph.D.dissertation,2002, University of California, Berkeley.

[50] D. Martin, C. Fowlkes, D. Tal, J. Malik, A database of human segmented natural images and its application to evaluating algorithms and measuring ecological statistics, ICCV (2001) 416-423.

[51] J. Freixenet, X. Munoz, D. Raba, J. Marti, and X. Cuff, "Yet Another Survey on Image Segmentation: Region and Boundary Information Integration," Proc. European Conf. Computer Vision, pp. 408-422, 2002.

[52] M. Meila, "Comparing Clusterings: An Axiomatic View," Proc. 22nd Int'l Conf. Machine Learning, pp. 577- 584, 2005

[53] R. Unnikrishnan, C. Pantofaru, and M. Hebert, "A Measure for Objective Evaluation of Image Segmentation Algorithms," Proc. CVPR Workshop Empirical Evaluation Methods in Computer Vision, 2005.

[54] L. Szilagri, Z. Benyo, S.M. Szilagyi, et al "MR Brain Image Segmentation Using an Enhanced Fuzzy C-Means Algorithm ", Proceedings of the 25th ,Annual International Conference of the IEEE EMBS, Cancun, Mexico, Sep.17-21, 2003:724-726.

[55] R. Moller., R. Zeipelt. "Automatic segmentation of 3D-MRI data using a genetic algorithm, Medical Imaging and Augmented Reality", 2001. Proceedings. International Workshop on, 10-12 June 2001:278 - 281.

[56] G. Gerig, J. Martin, R. Kikinis, O. Kubler, M. Shenton, F.A. Jolesz, Unsupervised tissue-type segmentation of 3-D dual-echo MR head data, Image and Vision Computing 10 (1992) 349. 
[57] M. Bomans, K.-H. HoÈ hne, U. Tiede, M. Riemer, 3-D segmentation of MR images of the head for 3-D display, IEEE Transactions on Medical Imaging 9 (1990) 177.

[58] H. Zhang, J. E. Fritts, Image segmentation evaluation: A survey of unsupervised methods, computer vision and image understanding 110 , (2008) 260-280.
[59] S.Rian, C. Jqggim J. Xue, 'Brain Tissue classification of magnetic Resonance images using partial volume modeling', IEEE Trans. On medical imaging, vol.19, No.2, pp.1179-1187, 2000.

[60] Database of image, www.cma.mgh.harvard.edu/ibsr. 